\title{
INVITED EDITORIAL Human Female Meiosis: New Insights into an Error-Prone Process
}

\author{
Dorothy Warburton
}

Departments of Genetics and Development and of Pediatrics, College of Physicians and Surgeons, Columbia University, New York

Meiosis is, of course, the process most fundamental to the science of genetics. As students we studied diagrams illustrating the various stages of meiosis, struggled with memorizing their difficult names, and learned how to relate these to Mendelian segregation. If we are old enough, we probably viewed-or even prepared-microscope slides of meiotic stages from organisms such as the grasshopper or Tradescentia, where it was possible to be convinced that things might actually happen the way that they were shown in the diagrams. After that point, most human geneticists take meiosis for granted. Nevertheless, human meiosis, at least in females, is a process that is highly error prone, a fact whose practical consequences keep clinical cytogenetic laboratories busy doing prenatal diagnosis. At least $5 \%$ of recognized human conceptions are aneuploid, and $4 \%$ have autosomal trisomy. In $>90 \%$ of cases this is the result of a maternal meiotic error, and, for all chromosomes except the largest, the error rate increases with maternal age (Hassold 1996). Given only the data on the proportion of aneuploidy in recognized embryonic and fetal deaths, one can estimate that $>20 \%$ of oocytes in women $>40$ years of age have failed to undergo meiosis correctly.

Most recent progress in our understanding of meiosis has been derived through the study of nonmammalian systems such as yeast and Drosophila, in which the traditional genetic approach of the analysis of mutations has led to significant advances in understanding recombination, synapse, and segregation. Because the meiotic chromosomes of these organisms are very difficult to resolve under the microscope, progress has usually involved the genetic analysis of the products of meiosis, rather than direct observations of the process, although there are a few heroic exceptions in which fluorescent microscopy has been used to observe chromosomal behavior directly (Demburg et al. 1996). Mammalian mei-

Received March 12, 1997; accepted for publication May 15, 1997. Address for correspondence and reprints: Dr. Dorothy Warburton, Genetics Diagnostic Laboratory, Babies Hospital, B-7, 3959 Broadway, New York, NY 10032-1537. E-mail: cuh@cuccfa.ccc.columbia .edu

This editorial represents the opinion of the author and has not been peer reviewed.

(C) 1997 by The American Society of Human Genetics. All rights reserved. 0002-9297/97/6101-0002\$02.00 osis has not been amenable to the same kind of genetic analysis, until quite recently, with $(a)$ the generation of mouse knockouts for homologues of nonmammalian meiotic genes and $(b)$ the discovery of meiotic effects of other genes. Thus, two genes (Mlh1 and Pms2) whose mutations lead to microsatellite instability in cancers, the ATM gene involved in ataxia-telangiectasia, and the BRCA1 gene have all been shown to have important functions in meiotic prophase. The elegant cytological observations of mouse and human meiosis, by Terry Ashley and others (Meyn et al. 1996; Plug et al. 1997; Scully et al. 1997), have been able to localize the products of these genes on meiotic chromosomes, suggesting functions in recombination and pairing for these and other genes, such as Rad51 and DNA polymerase beta.

Another approach to the genetic analysis of meiotic errors has been the use of highly polymorphic genetic markers to analyze segregation and recombination in trisomic human conceptions (Antonarakis et al. 1993; Robinson et al. 1993; Sherman et al. 1994; Hassold et al. 1995). From these studies came the first accurate estimates of the relative frequency of maternal and paternal errors, as well as data concerning recombination patterns on chromosomes undergoing nondisjunction. These data for trisomy 21 and chromosome 16 are now very robust, and they tell a story with both expected and unexpected twists (Lamb et al. 1996).

The working hypothesis of these investigations was that meiosis in humans would be similar to that in other organisms, in which meiotic nondisjunction and aneuploidy had been shown to occur as a result of decreased recombination. Errors classified as occurring in maternal meiosis I, because of the maintenance of maternal heterozygosity for markers near the centromere, behaved according to expectation. Recombination was reduced in the abnormally segregating chromosomes, especially near the centromere. Since the length of meiosis I in mammals is directly correlated with maternal age, it was also expected - and verified - that meiosis I errors were associated with increased maternal age. The data thus support the hypothesis that, in an older woman, bivalents lacking pericentromeric chiasmata separate prematurely, resulting in meiotic nondisjunction, whereas in a young woman the same oocyte is likely to be processed normally. 
Approximately one-quarter of cases of trisomy 21 are classified as meiosis II errors, because of homozygosity for maternally heterozygous pericentromeric markers. Because the length of meiosis II is unrelated to maternal age, the finding that these cases had a maternal age even greater than that of the meiosis I errors was unexpected. Furthermore, errors classified as occurring in meiosis II showed a much higher than normal rate of recombination in the pericentromeric region. Lamb et al. (1996) have proposed an explanation for these findings: bivalents with too much pericentromeric recombination fail to separate at meiosis I, with a subsequent reduction division of the bivalent occurring in meiosis II. The resulting meiotic products will be classified as meiosis II errors on the basis of their homozygosity for centromeric markers, even though the nondisjunction is actually due to an abnormal meiosis I division.

These data could then be interpreted to mean that almost all identified human trisomic conceptions result from errors in maternal meiosis I and that the nature of the factors leading to the maternal age effect is to add a "second hit" to bivalents with particular patterns of recombination. In the fetal oocyte one need only postulate a stochastic distribution of recombination, producing, at both ends of the distribution, oocytes that are susceptible to nondisjunction in older women. Of course, the nature of the factors that cause this increased susceptibility remain open to discovery.

Although an intriguing hypothesis, the relationship of differences in recombination among human bivalents to meiotic nondisjunction is currently supported only by circumstantial evidence. What kinds of more direct evidence might be used to support this hypothesis? One answer might come from the observation of the abnormal products observed in human oocytes undergoing meiosis. The technical difficulties of obtaining and analyzing human material had for a long time severely limited such studies. The critical steps of synapse and recombination occur in the fetal ovary, whereas the resumption of meiosis can be observed only in ovulated oocytes, normally only one per menstrual cycle. Although many fundamental questions can be studied in model organisms such as the mouse, extrapolation is limited by the fact that the error rate in human female meiosis differs, by an order of magnitude, from that found in other mammals that have been studied. Direct observation of human oocytes is therefore critical.

Since the advent of in vitro fertilization, a large number of studies have reported the chromosome complements of human oocytes retrieved for this purpose. The usual approach is to make use of oocytes that fail to be fertilized and to observe these when arrested in metaphase II. This should provide a direct answer to the question of the frequency and kinds of errors that have occurred in meiosis I. However, many of the earlier stud- ies relied on preparations of poor quality, in which accurate identification of chromosome groups or abnormalities was very difficult, and the method of spreading led to high rates of artifactual chromosome loss. Estimates of the frequency of abnormalities showed wide variability; the expected association of aneuploid gametes with maternal age could not be demonstrated; and the data failed to reproduce the expected distribution or frequency of human trisomies (Jacobs 1992). Angell (1991), using an improved method of preparation adapted from Mikamo and Yamaguchi (1983), first suggested that extra or missing chromatids, rather than univalents, might be the predominant error seen in human metaphase II oocytes - and that the preparations from other studies needed to be reinterpreted in the light of this finding.

The paper by Angell (1997) in this issue of the Journal reports on a much larger series of 200 analyzed metaphase II oocytes, confirming the previous findings. Surprisingly, no metaphase II oocytes were observed with additional whole univalents, as would be expected in meiosis I nondisjunction of the classical sort. However, a high proportion $(33 \%)$ showed prematurely separated chromatids (half-univalents), which could be extra $(23$ $+1 / 2)$, missing $(22+1 / 2)$, or balanced $(22+1 / 2+$ $1 / 2$ ). Premature equational division of the chromatids of univalents is hypothesized to occur as a result of premature separation of the bivalent in meiosis I. This hypothesis is supported by the observation of prematurely separated bivalents in a small sample of meiosis I oocytes. Adding credence to these data is (1) the predominance of abnormalities involving chromosome 16, which is by far the most common trisomy among recognized conceptions, and (2) the compatibility between the frequency and chromosomal distribution of observed oocyte abnormalities and that predicted from data on spontaneous abortions. Furthermore, oocytes with these chromatid anomalies occurred more often in older women.

Angell's (1997) paper thus confirms one of the predictions of the Lamb et al. (1996) model-that is, that premature separation of bivalents in meiosis I occurs in oocytes of older women. However, it does not confirm the second prediction, involving cases defined as meiosis II errors. True nondisjunction resulting from excessive pericentromeric recombination should lead to additional or missing whole univalents - or even to bivalents - at metaphase II, but no such errors were observed. Although "meiosis II" errors occur more rarely than meiosis I errors-and almost never for chromosome 16-some would have been expected among the 200 oocytes in Angell's series.

Other studies (Kamiguchi et al. 1993; Lim et al. 1995), using similar methods, have confirmed the presence of the unbalanced chromatid abnormalities first 
recognized by Angell, but they have also presented clear photographic evidence of oocytes with extra whole univalents. In Kamiguchi et al.'s (1993) study of 167 diploid oocytes, single chromatid anomalies were the sole abnormality in only 5 of 15 aneuploid oocytes (these authors did not include as abnormal those "balanced" chromatid anomalies that they considered to be technical artifacts). The overall frequency of aneuploid gametes in these other studies was quite similar to that in the study by Angell, even though the distribution of types of anomalies was different.

Another method of analyzing oocyte aneuploidy is the use of FISH with chromosome-specific centromere probes. Using probes that would identify abnormal segregation for chromosomes $13,18,21$, and X, Dailey et al. (1996) found evidence for an additional or missing univalent in 15 of 168 oocytes. They also confirmed the presence of premature chromatid separation, both balanced and unbalanced, but suggested that a large proportion of the balanced type may result from an in vitro effect dependent on time in culture. A strength of their study was the scoring of aneuploidy only when this was confirmed in both oocytes and polar body. Since they could examine only four chromosome pairs and were judging the type of abnormality only by the positions of the centromeres, their results are difficult to compare with those of other groups, who examined whole-chromosome preparations. However, statistical considerations suggest that the FISH technique may somehow have produced anomalous results. Dailey et al. (1996) identified $20 \%$ of oocytes as aneuploid, although they examined only four chromosome pairs-13, 18, 21 , and X. Since trisomies for these chromosomes constitute only approximately one-fifth of all trisomies in spontaneous abortions, an implausibly high proportion of oocytes would have to be aneuploid to account for the observed distribution of trisomies. A similar problem occurs in FISH studies of early embryos fertilized in vitro, in which an unexpectedly high proportion are scored as aneuploid for only a few chromosomes when FISH is used (Munné et al. 1995).

It is difficult to reconcile the differences in these oocyte analyses. Contributing factors may relate both to the study populations and to technical considerations. It is important to remember that all studies make use of less than optimal material. Women undergoing in vitro fertilization are not a random sample of women, since they clearly have problems leading to infertility. The hormonal environment leading to superovulation is also very different from that occurring in normal ovulation, and it is possible that variations in hormonal regimen account for some of the differences among studies. The oocytes available for study are those which failed in in vitro fertilization: any selective factors involved in this failure are totally unknown and, because of procedural differences, may again differ among in vitro-fertilization populations. All studies can use only a portion of the oocytes available for analysis, and the choice of which material to discard may affect the results. Studies also differ in the length of time that oocytes remained in culture. Since, at the moment, there would seem to be no way to avoid these problems with human oocyte material, a more accurate and consistent answer may have to await technical or medical developments that allow the study of more normal material.

Although direct observation of human oocyte meiosis is still technically very challenging, and although the results show puzzling inconsistencies, several facts do seem to emerge. First, there is clear evidence in metaphase II that premature separation of the bivalent in meiosis I is a major cause of aneuploidy. Most recent studies also agree that the frequency with which this occurs is related to maternal age. These facts are consistent with the hypothesis that oocytes with reduced recombination are less likely to be processed normally in older women. However, no studies of metaphase II oocytes have observed persistent bivalents, as would be predicted by Lamb et al.'s (1996) recent suggestion that errors scored as occurring in meiosis II result from disjunction of bivalents at meiosis II rather than at meiosis I.

The cytological observation of human oocyte meiosis may benefit from new approaches that take advantage of FISH to analyze oocyte behavior and that promise to yield interesting information. The previous difficulties of resolving and identifying individual chromosome pairs in the fetal oocyte, where the crucial stages of synapse and recombination occur, are now potentially solvable by use of chromosome- and locus-specific FISH probes. The work of Cheng and Gartler (1994; also see Cheng et al. 1995) has demonstrated the feasibility of using FISH to analyze the stages of meiotic prophase in both normal and abnormal human fetal oocytes. Techniques have also been developed in the mouse that permit preovulatory-mouse oocytes to complete meiosis in culture. Hunt et al. (1995) have demonstrated the utility of this approach for observing meiosis in cytogenetically normal and abnormal mice. This and studies in other organisms suggest that the high aneuploidy rate in oocytes may reflect a lack of the same strong checkpoint mechanism to inhibit meiosis in the presence of unpaired chromosomal elements that is present in male mammalian meiosis. Similar studies on human oocytes might be very revealing if suitable material were available.

\section{References}

Angell RR (1991) Predivision in human oocytes at meiosis I: a mechanism for trisomy formation in man. Hum Genet 86 : 383-387 
(1997) First-meiotic-division nondisjunction in human oocytes. Am J Hum Genet 61:23-32 (in this issue)

Antonarakis SE, Avramopolous D, Blouin J-L, Talbot CC, Schinzel AA (1993) Mitotic errors in somatic cells cause trisomy 21 in $4.5 \%$ of cases and are not associated with advanced maternal age. Nat Genet 3:146-150

Cheng EY, Chen YJ, Gartler SM (1995) Chromosome painting analysis of early oogenesis in human trisomy 18 . Cytogenet Cell Genet 70:205-210

Cheng EY, Gartler SM (1994) A fluorescent in situ hybridization analysis of $\mathrm{X}$ chromosome pairing in early human female meiosis. Hum Genet 94:389-394

Dailey T, Dale B, Cohen J, Munné S (1996) Association between nondisjunction and maternal age in meiosis II human oocytes. Am J Hum Genet 59:176-184

Demburg AF, Sedat JW, Hawley RS (1996) Direct evidence of a role for heterochromatin in meiotic chromosome segregation. Cell 86:135-146

Hassold TJ (1996) Human aneuploidy: incidence, origin and etiology. Environ Mol Mutagen 28:167-175

Hassold T, Merrill M, Adkins K, Freeman S, Sherman S (1995) Recombination and maternal age-dependent nondisjunction: molecular studies of trisomy 16 . Am J Hum Genet 57: 867-874

Hunt P, LeMaire R, Embury P, Sheean L, Mroz K (1995) Analysis of chromosome behavior in intact mammalian oocytes. Hum Mol Genet 4:2007-2012

Jacobs P (1992) The chromosome complement of human gametes. Oxf Rev Reprod Biol 14:48-72

Kamiguchi Y, Rosenbusch B, Sterizk K, Mikamo K (1993) Chromosome analysis of unfertilized human oocytes prepared by a gradual fixation-air drying method. Hum Genet 90:533-541

Lamb NE, Freeman SB, Savage-Austin A, Pettay D, Taft L,
Hersey J, Gu Y, et al (1996) Susceptible chiasmate configurations of chromosome 21 predispose to non-disjunction in both maternal meiosis I and meiosis II. Nat Genet 14:400405

Lim A, Ho A, Tsakok M (1995) Chromosomes of oocytes failing in vitro fertilization. Hum Reprod 10:2570-2575

Meyn MS, Moss SB, Carr AM, Ashley T, Heidstra MF (1996) The Atr and Atm protein kinases associate with different sites along meiotically pairing chromosomes. Genes Dev 10: 2423-2437

Mikamo K, Kamiguchi Y (1983) A new assessment system for chromosomal mutagenicity using oocytes and early zygotes in the Chinese hamster. In: Ishihara T, Sasaki MS (eds) Radiation-induced chromosome damage in man. Alan $\mathbf{R}$ Liss, New York, pp 411-432

Munne S, Alikani M, Tomkin G, Grifo J, Cohen J (1995) Embryo morphology, developmental rates and maternal age are correlated with chromosome abnormalities. Fertil Steril 64:382-391

Plug AW, Clairmont CA, Sapi E, Ashley T, Sweasy JB (1997) Evidence for a role for DNA polymerase beta in mammalian meiosis. Proc Natl Acad Sci USA 94:1327-1231

Robinson WP, Bernasconi F, Mutirangura A, Ledbetter DH, Langlois S, Malcolm S, Morris MA, et al (1993) Nondisjunction of chromosome 15: origin and recombination. Am J Hum Genet 53:740-751

Scully R, Chen J, Plug A, Eiao Y, Feunteun J, Ashley T, Livingston DM (1997) Association of BRCA1 with Rad51 in mitotic and meiotic cells. Cell 24:265-275

Sherman SL, Petersen MB, Freeman SB, Hersey J, Pettay D, Taft L, Frantzen M, et al (1994) Nondisjunction of chromosome 21 in maternal meiosis I: evidence for a maternal-age dependent mechanism involving reduced recombination. Hum Mol Genet 3:1529-1535 